\title{
Group Lifting Structures for Multirate Filter Banks, II: Linear Phase Filter Banks
}

\author{
Christopher M. Brislawn \\ Los Alamos National Laboratory, MS B265, Los Alamos, NM 87545-1663 USA \\ (505) 665-1165 (office); (505) 665-5220 (FAX); e-mail: brislawn@lanl.gov
}

\section{Final Revision, Reformatted for arXiv.org}

\begin{abstract}
The theory of group lifting structures is applied to linear phase lifting factorizations for the two nontrivial classes of two-channel linear phase perfect reconstruction filter banks, the whole- and halfsample symmetric classes. Group lifting structures defined for the reversible and irreversible classes of whole- and half-sample symmetric filter banks are shown to satisfy the hypotheses of the uniqueness theorem for group lifting structures. It follows that linear phase group lifting factorizations of wholeand half-sample symmetric filter banks are therefore independent of the factorization methods used to construct them. These results cover the specification of whole-sample symmetric filter banks in the ISO/IEC JPEG 2000 image coding standard.
\end{abstract}

\section{Index Terms}

Filter bank, wavelet, unique factorization, polyphase, lifting, linear phase filter, group.

The author is with Los Alamos National Laboratory, Los Alamos, NM 87545-1663 USA (e-mail: brislawn@lanl.gov). Los Alamos National Laboratory is operated by Los Alamos National Security LLC for the U. S. Department of Energy under contract DE-AC52-06NA25396. This work was supported by the Los Alamos Laboratory-Directed Research \& Development Program. 
CONTENTS

I Introduction 3

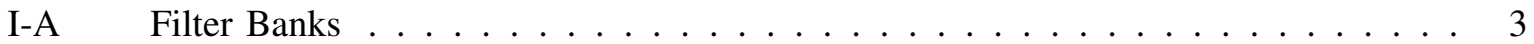

I-B Lifting Factorizations $\ldots \ldots \ldots \ldots \ldots \ldots$

I-C Group Lifting Structures $\ldots \ldots \ldots \ldots$

II Sufficient Conditions for Order-Increasing Group Lifting Structures 6

III Whole-Sample Symmetric Filter Banks 8

III-A Main Uniqueness Result . . . . . . . . . . . . . . . . . . . . . 8

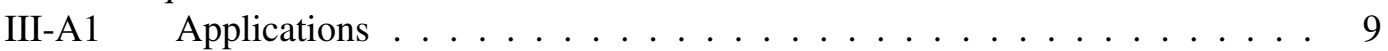

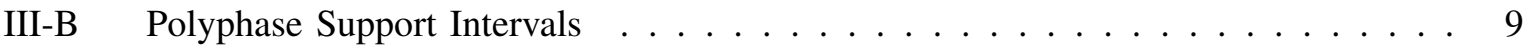

III-C The WS Support Interval Formula . . . . . . . . . . . . . . . . 11

III-D The Support-Covering Property . . . . . . . . . . . . . . . . . . . . 14

IV Half-Sample Symmetric Filter Banks

IV-A Main Uniqueness Result . . . . . . . . . . . . . . . . . 16

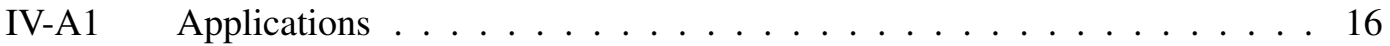

IV-B Polyphase Support Intervals . . . . . . . . . . . . . . . . . . 17

IV-C The HS Support Interval Formula . . . . . . . . . . . . . . . . . . 18

IV-D The Support-Covering Property . . . . . . . . . . . . . . . . 20

V Conclusions $\quad 21$

References 


\section{INTRODUCTION}

This is the second paper on a new approach to lifting factorization for two-channel FIR perfect reconstruction filter banks. The first paper [1] introduced group lifting structures to parameterize universes of lifting factorizations for given classes of filter banks. While lifting factorizations are generally nonunique, it was proven in [1, Theorem 1] that, under suitable hypotheses, a filter bank has a unique irreducible lifting factorization within a given group lifting structure. The present paper applies [1, Theorem 1] to group lifting structures for linear phase lifting factorizations of whole-sample symmetric (WS) and halfsample symmetric (HS) filter banks. Existence of linear phase lifting factorizations for these classes was proven in [2], and group lifting structures were introduced in [1, Section IV] for both irreversible and reversible factorizations. Using [1, Theorem 1], we now prove uniqueness results for irreducible group lifting factorizations of these filter banks.

For WS filter banks, like those in the ISO/IEC JPEG 2000 standard [3, Annex F], [4, Annex G], our results imply there is only one way to factor them into alternating upper and lower triangular lifting matrices with HS lifting filters. Lifting HS filter banks from concentric, equal-length HS base filter banks with whole-sample antisymmetric (WA) lifting filters [2] is shown to be unique modulo one trivial degree of freedom ("unique modulo rescaling"). The key to [1, Theorem 1] is verifying a polyphase order-increasing hypothesis [1, Definition 10] by computing the order of lifted filter banks.

The remainder of the Introduction reviews key notation and concepts from [1], adding some useful computational tools. Section II proves a technical result, Lemma 2, that gives sufficient conditions for a group lifting structure to satisfy the order-increasing hypothesis. Section III uses Lemma 2 to prove that the reversible and irreversible WS group lifting structures satisfy the order-increasing property and generate unique group lifting factorizations. Analogous results for HS group lifting structures are proven in Section IV] Section V] contains concluding remarks.

\section{A. Filter Banks}

Given an FIR filter,

$$
F(z)=\sum_{n=a}^{b} f(n) z^{-n},
$$

the support interval of $f$ is defined [1, Definition 1] to be the largest closed interval of integers for which $f(a), f(b) \neq 0$ :

$$
\operatorname{supp\_ int}(F) \equiv \operatorname{supp} \_\operatorname{int}(f) \equiv[a, b] \subset \mathbb{Z} .
$$

If supp_int $(f)=[a, b]$ then the order of $F$ is defined to be

$$
\operatorname{order}(F) \equiv b-a \text {. }
$$

Define the filter's support radius to be the integer

$$
\operatorname{supp\_ rad}(f) \equiv\left\lfloor\frac{b-a+1}{2}\right\rfloor \text {. }
$$

The polyphase vector representation of an FIR filter,

$$
\begin{aligned}
\boldsymbol{F}(z) & \equiv\left[\begin{array}{l}
F_{0}(z) \\
F_{1}(z)
\end{array}\right]=\sum_{n=c}^{d} \boldsymbol{f}(n) z^{-n}, \\
\boldsymbol{f}(n) & \equiv\left[\begin{array}{l}
f_{0}(n) \\
f_{1}(n)
\end{array}\right] \quad \text { with } \boldsymbol{f}(c), \boldsymbol{f}(d) \neq \mathbf{0},
\end{aligned}
$$


is defined in [1, Section II-A] based on the analysis polyphase-with-advance representation of the scalar filter [2],

$$
F(z)=F_{0}\left(z^{2}\right)+z F_{1}\left(z^{2}\right) .
$$

The polyphase support interval of the filter with polyphase vector representation (5) is defined in [1] to be

$$
\operatorname{supp} \_\operatorname{int}(\boldsymbol{f}) \equiv[c, d] \text {. }
$$

Define the join of two closed intervals, denoted with a $\vee$, to be the smallest closed interval containing their union:

$$
[a, b] \vee[c, d] \equiv[\min (a, c), \max (b, d)] .
$$

It is straightforward to verify that the polyphase support interval 6 is given by the join of the scalar support intervals for the two polyphase component filters:

$$
\operatorname{supp} \_i n t(\boldsymbol{f})=\operatorname{supp} \_\operatorname{int}\left(f_{0}\right) \vee \operatorname{supp} \_\operatorname{int}\left(f_{1}\right) \text {. }
$$

Similarly, consider an FIR filter bank with polyphase matrix

$$
\begin{aligned}
& \mathbf{H}(z) \equiv\left[\begin{array}{l}
\boldsymbol{H}_{0}^{T}(z) \\
\boldsymbol{H}_{1}^{T}(z)
\end{array}\right]=\sum_{n=c}^{d} \mathbf{h}(n) z^{-n}, \\
& \mathbf{h}(n) \equiv\left[\begin{array}{l}
\boldsymbol{h}_{0}^{T}(n) \\
\boldsymbol{h}_{1}^{T}(n)
\end{array}\right] \quad \text { with } \mathbf{h}(c), \mathbf{h}(d) \neq \mathbf{0} .
\end{aligned}
$$

The polyphase support interval of the filter bank 9,10 ) is defined in [1, Section II-A] to be supp_int $(\mathbf{h}) \equiv$ $[c, d]$. Again, it is straightforward to verify that the polyphase support interval of the filter bank is equal to the join of the polyphase support intervals of the two polyphase filter vectors:

$$
\operatorname{supp} \_\operatorname{int}(\mathbf{h})=\operatorname{supp} \_i n t\left(\boldsymbol{h}_{0}\right) \vee \operatorname{supp} \_\operatorname{int}\left(\boldsymbol{h}_{1}\right) \text {. }
$$

The following lemma describes the support intervals that result from performing elementary operations on FIR filters.

Lemma 1: If $F(z)$ and $G(z)$ are scalar FIR filters then

$$
\operatorname{supp} \_\operatorname{int}(f * g)=\operatorname{supp} \_\operatorname{int}(f)+\operatorname{supp} \_ \text {int }(g) \text {. }
$$

(Note that (12) does not extend to matrix-vector or matrix-matrix convolution; the lack of such a simple formula for matrix-matrix convolution is responsible for many of the technical difficulties in this paper.) If $\mathbf{F}(z)$ is a matrix-, vector-, or scalar-valued FIR filter with supp_int $(\mathbf{f})=[a, b]$ then

$$
\text { supp_int }\left(\mathbf{F}\left(z^{2}\right)\right)=[2 a, 2 b] \text {. }
$$

If $\mathbf{G}(z)$ has the same dimensions as $\mathbf{F}(z)$ and supp_int $(\mathbf{g}) \subset(a, b)=[a+1, b-1]$, where $\subset$ denotes (non-proper) containment with possible equality, then

$$
\operatorname{supp} \_i n t(\mathbf{f}+\mathbf{g})=\operatorname{supp} \_\operatorname{int}(\mathbf{f}) \text {. }
$$




\section{B. Lifting Factorizations}

Part I [1] works with partially factored lifting cascades,

$$
\mathbf{H}(z)=\operatorname{diag}(1 / K, K) \mathbf{S}_{N-1}(z) \cdots \mathbf{S}_{0}(z) \mathbf{B}(z),
$$

relative to a base filter bank, $\mathbf{B}(z)$. Cascades that are completely factored into lifting steps correspond to $\mathbf{B}(z)=\mathbf{I}$. The update characteristic, $m_{n}$, of $\mathbf{S}_{n}(z)$ [1, Definition 2] indicates whether a lifting matrix is upper triangular (i.e., a lowpass update) or lower triangular (a highpass update):

$$
m_{n}= \begin{cases}0 & \text { if } \mathbf{S}_{n}(z) \text { is upper triangular, } \\ 1 & \text { if } \mathbf{S}_{n}(z) \text { is lower triangular. }\end{cases}
$$

A lifting cascade is irreducible [1, Definition 3] if the lifting steps strictly alternate between lower and upper triangular, implying that $m_{n+1}=1-m_{n}$ for irreducible liftings.

Many of the arguments in this paper involve finite induction and are based on the recursive formulation of lifting:

$$
\begin{aligned}
\mathbf{H}(z) & =\operatorname{diag}(1 / K, K) \mathbf{E}^{(N-1)}(z), \\
\mathbf{E}^{(n)}(z) & =\mathbf{S}_{n}(z) \mathbf{E}^{(n-1)}(z) \quad \text { for } 0 \leq n<N, \\
\mathbf{E}^{(-1)}(z) & \equiv \mathbf{B}(z) .
\end{aligned}
$$

The scalar filters corresponding to an intermediate partial product of lifting matrices are given by [2, eqn. (9)],

$$
\left[\begin{array}{l}
E_{0}^{(n)}(z) \\
E_{1}^{(n)}(z)
\end{array}\right]=\mathbf{E}^{(n)}\left(z^{2}\right)\left[\begin{array}{l}
1 \\
z
\end{array}\right]=\mathbf{S}_{n}\left(z^{2}\right)\left[\begin{array}{l}
E_{0}^{(n-1)}(z) \\
E_{1}^{(n-1)}(z)
\end{array}\right],
$$

where $E_{i}^{(-1)}(z) \equiv B_{i}(z)$ for $i=0,1$. We make extensive use of the scalar version of this recursion, based on the update characteristic, $m_{n}$, of $\mathbf{S}_{n}(z)$. The filter updated by $\mathbf{S}_{n}(z)$ is $E_{m_{n}}^{(n)}(z)$, while $E_{1-m_{n}}^{(n)}(z)$ is not modified by $\mathbf{S}_{n}(z)$. Thus, 18 corresponds to the scalar lifting formulas

$$
\begin{aligned}
E_{m_{n}}^{(n)}(z) & =E_{m_{n}}^{(n-1)}(z)+S_{n}\left(z^{2}\right) E_{1-m_{n}}^{(n-1)}(z), \\
E_{1-m_{n}}^{(n)}(z) & =E_{1-m_{n}}^{(n-1)}(z) .
\end{aligned}
$$

\section{Group Lifting Structures}

A group lifting structure, $\mathfrak{S}$, is defined in [1, Definition 6] to be an ordered four-tuple,

$$
\mathfrak{S} \equiv(\mathcal{D}, \mathcal{U}, \mathcal{L}, \mathfrak{B})
$$

$\mathcal{D}$ is an abelian group of diagonal gain scaling matrices, $\mathbf{D}_{K} \equiv \operatorname{diag}(1 / K, K) . \mathcal{U}$ and $\mathcal{L}$ are abelian groups of upper and lower triangular lifting matrices, and $\mathfrak{B}$ is a set of base filter banks. The lifting cascade group, $\mathcal{C}$, is the nonabelian matrix group generated by $\mathcal{U}$ and $\mathcal{L}$,

$$
\mathcal{C} \equiv\langle\mathcal{U} \cup \mathcal{L}\rangle=\left\{\mathbf{S}_{1} \cdots \mathbf{S}_{k}: k \geq 1, \mathbf{S}_{i} \in \mathcal{U} \cup \mathcal{L}\right\}
$$

The universe of all lifted filter banks generated by $\mathfrak{S}$ is

$$
\mathcal{D} \mathcal{C} B\{\mathbf{D C B}: \mathbf{D} \in \mathcal{D}, \mathbf{C} \in \mathcal{C}, \mathbf{B} \in \mathfrak{B}\} \text {. }
$$

The matrix $\mathbf{D}_{K} \in \mathcal{D}$ acts on transfer matrices via the inner automorphism $\gamma_{K}$,

$$
\gamma_{K} \mathbf{A}(z) \equiv \mathbf{D}_{K} \mathbf{A}(z) \mathbf{D}_{K}^{-1} .
$$


A group $\mathcal{G}$ of transfer matrices is $\mathcal{D}$-invariant [1, Definition 5] if $\gamma_{K} \mathcal{G}=\mathcal{G}$ for all $\mathbf{D}_{K} \in \mathcal{D}$, and a group lifting structure $(21)$ is $\mathcal{D}$-invariant if $\mathcal{U}$ and $\mathcal{L}$, and therefore $\mathcal{C}$, are $\mathcal{D}$-invariant. $\mathcal{D}$-invariance is easy to verify in practice using [1, Lemma 2].

A lifting cascade (15] is called strictly polyphase order-increasing (or just order-increasing) [1, Definition 10] if the intermediate partial products (17) satisfy

$$
\operatorname{order}\left(\mathbf{E}^{(n)}\right)>\operatorname{order}\left(\mathbf{E}^{(n-1)}\right) \quad \text { for } 0 \leq n<N .
$$

A group lifting structure, $\mathfrak{S}$, is called order-increasing if every irreducible cascade in $\mathfrak{C} \mathfrak{B}$ is orderincreasing.

If $\mathfrak{S}$ is an order-increasing, $\mathcal{D}$-invariant group lifting structure, the uniqueness theorem [1, Theorem 1] says that all irreducible factorizations of $\mathbf{H}(z)$ in $\mathfrak{S}$ are "equivalent modulo rescaling." Specifically, given two such factorizations,

$$
\begin{aligned}
\mathbf{H}(z) & =\mathbf{D}_{K} \mathbf{S}_{N-1}(z) \cdots \mathbf{S}_{0}(z) \mathbf{B}(z) \\
& =\mathbf{D}_{K^{\prime}} \mathbf{S}_{N^{\prime}-1}^{\prime}(z) \cdots \mathbf{S}_{0}^{\prime}(z) \mathbf{B}^{\prime}(z),
\end{aligned}
$$

the uniqueness theorem states that the number of lifting steps is the same $\left(N^{\prime}=N\right)$, with base filter banks related by

$$
\mathbf{B}^{\prime}(z)=\mathbf{D}_{\alpha} \mathbf{B}(z) \text { for constant } \alpha=K / K^{\prime},
$$

and lifting steps related by inner automorphisms,

$$
\mathbf{S}_{i}^{\prime}(z)=\gamma_{\alpha} \mathbf{S}_{i}(z), \quad i=0, \ldots, N-1 .
$$

We express this by saying that irreducible lifting factorizations in $\mathfrak{S}$ are "unique modulo rescaling" [1, Definition 11].

The theorem can be strengthened to yield unique irreducible factorizations by normalizing a degree of freedom in the base filter banks. For instance, fixing a lowpass DC response like $B_{0}(1)=1$ for all $\mathbf{B}(z) \in \mathfrak{B}$ implies $\alpha=1$ by 25 .

Our principal tool for verifying the order-increasing hypothesis of [1, Theorem 1] is developed in the next section.

\section{SUfFICIENT CONDITIONS FOR ORDER-INCREASING GROUP LifTING STRUCTURES}

Since lifting only modifies one filter at a time, the order-increasing property hinges on the growth of the support intervals of the filters $E_{m_{n}}^{(n)}(z)$ being updated in each lifting step. A simplification occurs in the WS and HS cases because, as we shall show, the polyphase support interval of the updated polyphase filter vector, $\boldsymbol{E}_{m_{n}}^{(n)}(z)$, always contains that of its complement, $\boldsymbol{E}_{1-m_{n}}^{(n)}(z)$, making the polyphase order of $\mathbf{E}^{(n)}(z)$ equal to the polyphase order of $\boldsymbol{E}_{m_{n}}^{(n)}(z)$. This is convenient, but other order-increasing group lifting structures may exist that do not share this property.

Lemma 2: Let $\mathfrak{S}$ be a group lifting structure satisfying the following two polyphase vector conditions.

1) For all $\mathbf{B}(z) \in \mathfrak{B}$, the polyphase support intervals $(6)$ for the base polyphase filter vectors are equal:

$$
\operatorname{supp} \_ \text {int }\left(\boldsymbol{b}_{0}\right)=\operatorname{supp} \_ \text {int }\left(\boldsymbol{b}_{1}\right) \text {. }
$$

2) For all irreducible lifting cascades in $\mathcal{C} \mathfrak{B}$, the polyphase support intervals $(6)$ for the intermediate polyphase filter vectors satisfy the proper inclusions

$$
\operatorname{supp} \_ \text {int }\left(\boldsymbol{e}_{1-m_{n}}^{(n)}\right) \varsubsetneqq \operatorname{supp} \_ \text {int }\left(\boldsymbol{e}_{m_{n}}^{(n)}\right) \text { for } n \geq 0 .
$$


It then follows that $\mathfrak{S}$ is strictly polyphase order-increasing.

Proof: Let $\mathbf{S}_{N-1}(z) \cdots \mathbf{S}_{0}(z) \mathbf{B}(z) \in \mathcal{C} \mathfrak{B}$ be irreducible. By $\sqrt{11}$ and 27 the base filter bank satisfies

$$
\begin{aligned}
\operatorname{supp} \_i n t(\mathbf{b}) & =\operatorname{supp} \_\operatorname{int}\left(\boldsymbol{b}_{0}\right) \vee \operatorname{supp} \_i n t\left(\boldsymbol{b}_{1}\right) \\
& =\operatorname{supp} \_\operatorname{int}\left(\boldsymbol{b}_{0}\right) \\
& =\operatorname{supp} \_\operatorname{int}\left(\boldsymbol{b}_{1}\right)
\end{aligned}
$$

which implies

$$
\operatorname{order}(\mathbf{B})=\operatorname{order}\left(\boldsymbol{B}_{0}\right)=\operatorname{order}\left(\boldsymbol{B}_{1}\right)
$$

Similarly, (11) and 28] imply

$$
\begin{aligned}
\operatorname{supp} \_i n t\left(\mathbf{e}^{(n)}\right) & =\operatorname{supp} \_i n t\left(\boldsymbol{e}_{0}^{(n)}\right) \vee \operatorname{supp} \_i n t\left(\boldsymbol{e}_{1}^{(n)}\right) \\
& =\operatorname{supp} \_i n t\left(\boldsymbol{e}_{m_{n}}^{(n)}\right)
\end{aligned}
$$

and therefore

$$
\operatorname{order}\left(\mathbf{E}^{(n)}\right)=\operatorname{order}\left(\boldsymbol{E}_{m_{n}}^{(n)}\right)
$$

Note that 28$)$ also implies

$$
\operatorname{order}\left(\boldsymbol{E}_{m_{n}}^{(n)}\right)>\operatorname{order}\left(\boldsymbol{E}_{1-m_{n}}^{(n)}\right) \quad \text { for } n \geq 0
$$

We now show that order $\left(\mathbf{E}^{(n)}\right)$ is strictly increasing. we get

Case: $n=0$. Since the filter not being lifted is $\boldsymbol{E}_{1-m_{0}}^{(0)}(z)=\boldsymbol{B}_{1-m_{0}}(z)$, by 30, 31, and 29,

$$
\begin{aligned}
& \operatorname{order}\left(\mathbf{E}^{(0)}\right)=\operatorname{order}\left(\boldsymbol{E}_{m_{0}}^{(0)}\right) \\
& \quad>\operatorname{order}\left(\boldsymbol{E}_{1-m_{0}}^{(0)}\right)=\operatorname{order}\left(\boldsymbol{B}_{1-m_{0}}\right)=\operatorname{order}(\mathbf{B}) .
\end{aligned}
$$

Case: $n>0$. Since the cascade is irreducible by hypothesis, the filter not being lifted in step $n$ must have been lifted in step $n-1$ :

$$
\boldsymbol{E}_{1-m_{n}}^{(n)}(z)=\boldsymbol{E}_{m_{n-1}}^{(n-1)}(z)
$$

Therefore,

$$
\begin{aligned}
& \operatorname{order}\left(\mathbf{E}^{(n)}\right)=\operatorname{order}\left(\boldsymbol{E}_{m_{n}}^{(n)}\right) \\
& \quad>\operatorname{order}\left(\boldsymbol{E}_{1-m_{n}}^{(n)}\right)=\operatorname{order}\left(\boldsymbol{E}_{m_{n-1}}^{(n-1)}\right)=\operatorname{order}\left(\mathbf{E}^{(n-1)}\right)
\end{aligned}
$$


Remarks: The polyphase representation of the "causal lazy wavelet" filter bank [1, Example 7] does not satisfy (27). A scalar analogue of (28) became evident while computing linear phase lifting factorizations of both WS and HS filter banks, but the author found that (27) was the less-than-obvious key to unifying the WS and HS cases. The base filters,

$$
B_{0}(z)=1 \text { and } B_{1}(z)=z,
$$

for the (anticausal) lazy wavelet filter bank $\mathbf{B}(z)=\mathbf{I}$ have equal lengths but are not concentric like, e.g., the Haar filters,

$$
H_{0}(z)=(z+1) / 2 \quad \text { and } \quad H_{1}(z)=z-1 .
$$

An equal-length hypothesis for the base filters, though, is not enough by itself to imply the order-increasing property. Fortunately, (32) and (33) share a common feature that implies the order-increasing property, namely, the fact that in both cases the base filters have the same polyphase support intervals.

\section{Whole-S Ample Symmetric Filter BANKS}

We briefly review the definitions from [1, Section IV-A] of the group lifting structures for WS filter banks. For the irreversible WS group, $\mathcal{W}$, the upper (respectively, lower) triangular lifting matrices, $\mathcal{U} \equiv v\left(\mathcal{P}_{0}\right)$ (respectively, $\mathcal{L} \equiv \lambda\left(\mathcal{P}_{1}\right)$ ), are defined by half-sample symmetric real Laurent polynomials:

$$
\begin{aligned}
& \mathcal{P}_{0} \equiv\left\{S(z) \in \mathbb{R}\left[z, z^{-1}\right]: S\left(z^{-1}\right)=z S(z)\right\}, \\
& \mathcal{P}_{1} \equiv\left\{S(z) \in \mathbb{R}\left[z, z^{-1}\right]: S\left(z^{-1}\right)=z^{-1} S(z)\right\} .
\end{aligned}
$$

The scaling matrices are $\mathcal{D} \equiv\left\{\mathbf{D}_{K}: K \neq 0\right\}$ and the base filter banks are trivial: $\mathfrak{B} \equiv\{\mathbf{I}\}$. The irreversible WS group lifting structure is $\mathfrak{S}_{\mathcal{W}} \equiv(\mathcal{D}, \mathcal{U}, \mathcal{L}, \mathfrak{B})$, and its lifting cascade group is $\mathfrak{C}_{\mathcal{W}} \equiv\langle\mathcal{U} \cup \mathcal{L}\rangle$.

In the reversible case there are no scaling matrices, $\mathcal{D}_{r} \equiv\{\mathbf{I}\}$, the set of base filter banks is still $\mathfrak{B}_{r} \equiv\{\mathbf{I}\}$, and the lifting filters 34 , , 35 are restricted to those with dyadic rational coefficients. The reversible WS group is defined by

$$
\mathcal{W}_{r} \equiv \mathcal{C}_{\mathcal{W}_{r}} \equiv\left\langle\mathcal{U}_{r} \cup \mathcal{L}_{r}\right\rangle \text {, }
$$

and its group lifting structure is $\mathfrak{S}_{\mathcal{W}_{r}} \equiv\left(\mathcal{D}_{r}, \mathcal{U}_{r}, \mathcal{L}_{r}, \mathfrak{B}_{r}\right)$.

\section{A. Main Uniqueness Result}

Our main result on WS filter banks shows that $\mathfrak{S}_{\mathcal{W}}$ and $\mathfrak{S}_{\mathcal{W}_{r}}$ produce unique irreducible group lifting factorizations.

Theorem 1 (Uniqueness of WS group lifting factorizations): Let $\mathfrak{S}_{\mathcal{W}}$ and $\mathfrak{S}_{\mathcal{W}_{r}}$ be the group lifting structures defined in [1, Section IV-A]. Every filter bank in $\mathcal{W}$ has a unique irreducible lifting factorization in $\mathfrak{S}_{\mathcal{W}}$ and every filter bank in $\mathcal{W}_{r}$ has a unique irreducible lifting factorization in $\mathfrak{S}_{\mathcal{W}_{r}}$.

Proof: Existence of irreducible WS group lifting factorizations for these groups was covered in [1, Section IV-A].

Uniqueness: As discussed above, $\mathfrak{B}=\{\mathbf{I}\}$ satisfies hypothesis 27] in Lemma 2 since

$$
\operatorname{supp\_ int}\left(\boldsymbol{b}_{i}\right)=\{0\}, \quad i=0,1 .
$$

Lemma 6 (Section III-D below) shows that irreducible lifting cascades in $\mathcal{C}_{\mathcal{W}} \mathfrak{B}$ satisfy [28), so Lemma 2 implies that $\mathfrak{S}_{\mathcal{W}}$ is order-increasing. $\mathcal{D}$-invariance of $\mathcal{U}$ and $\mathcal{L}$ follows from [1, Lemma 2], so [1, Theorem 1] applies to $\mathfrak{S}_{\mathcal{W}}$. Since $\mathfrak{B}=\{\mathbf{I}\}$, it follows that the degree of freedom, $\alpha$, in the conclusion of [1, Theorem 1] must be unity: $\alpha=1$. This means that irreducible group lifting factorizations in $\mathfrak{S}_{\mathcal{W}}$ are unique.

In the reversible case we have $\mathcal{C}_{\mathcal{W}_{r}}<\mathcal{C}_{\mathcal{W}}$ and $\mathfrak{B}_{r}=\mathfrak{B}$. The reversible WS group lifting factorizations are thus a subset of the irreversible factorizations, $\mathcal{C}_{\mathcal{W}_{r}} \mathfrak{B}_{r} \subset \mathcal{D} \mathcal{C}_{\mathcal{W}} \mathfrak{B}$, so uniqueness of irreducible factorizations in $\mathfrak{S}_{\mathcal{W}_{r}}$ follows from uniqueness of irreducible factorizations in $\mathfrak{S}_{\mathcal{W}}$. 
Remarks: While existence of cascade factorizations for arbitrary WS filter banks was asserted for the cascade structure in [5], Theorem 11 provides a more complete theory of both existence and uniqueness for factorizations of WS filter banks.

1) Applications: Theorem 1 implies that compliant lifting factorizations of user-defined WS filter banks in JPEG 2000 Part 2, which are specified in [4, Annex G] as irreducible WS group lifting factorizations, are unique.

Corollary 1: A delay-minimized unimodular WS filter bank can be specified in JPEG 2000 Part 2 Annex G syntax in one and only one way.

Example 1: In their book on JPEG 2000 [6, Section 6.4.4], Taubman and Marcellin note the relative simplicity of implementing lifting factorizations that only involve HS lifting filters of length two. (It is clear from [6, Figure 6.13] that this means first-order filters; i.e., symmetric filters with two consecutive terms, such as $c\left(1+z^{-1}\right)$.) They then claim [6, page 294]:

All two channel FIR subband transforms having odd length, symmetric filters with least dissimilar lengths (filter lengths differ by 2 ) may be factored into lifting steps of this form [i.e., first-order HS lifting filters].

As an application of Theorem 1, we can construct counterexamples to this claim. Consider the following lifting cascade:

$$
\mathbf{H}(z)=\left[\begin{array}{cc}
1 & 1+z^{-1} \\
0 & 1
\end{array}\right]\left[\begin{array}{cc}
1 & 0 \\
z^{2}+z+1+z^{-1} & 1
\end{array}\right] .
$$

By (18) the corresponding WS scalar filter bank is

$$
\begin{aligned}
& H_{0}(z)=z^{4}+2 z^{2}+z+3+z^{-1}+2 z^{-2}+z^{-4} \\
& H_{1}(z)=z^{4}+z^{2}+z+1+z^{-2}
\end{aligned}
$$

which has filters of least dissimilar lengths. Theorem 1 implies that 36 is its unique irreducible WS group lifting factorization, so it cannot have another such factorization using first-order HS filters. Generalizing this example, it follows from Lemma 5 (below) that an irreducible WS group lifting cascade corresponds to a WS filter bank with filters of least dissimilar lengths if and only if the final HS lifting filter is first-order.

The lemmas needed to verify hypothesis 28 , of Lemma 2 occupy the remainder of Section III.

\section{B. Polyphase Support Intervals}

The relation between a filter's impulse response, $e(k)$, and its polyphase components, $e_{j}(k)$, is given in [2, eqn. (10)]:

$$
e_{j}(k)=e(2 k-j), \quad j=0,1, \quad k \in \mathbb{Z} .
$$

In general, the support intervals (1) for the individual polyphase components are not completely determined by supp_int $(e)$ (see the proof of the following lemma). Nonetheless, the polyphase support interval, supp_int $(\boldsymbol{e})$, is completely determined by supp_int $(e)$. The following derivations do not depend on linear phase properties of the filters.

Lemma 3: Suppose $E(z)$ is an odd-length FIR filter whose support interval (1) is centered at 0,

$$
\operatorname{supp\_ int}(e)=[-r, r],
$$

where $r \equiv \operatorname{supp\_ rad}(e) \geq 0$ is the support radius $(3)$. Then the polyphase support interval (6) of the filter is:

$$
\operatorname{supp\_ int}(\boldsymbol{e})= \begin{cases}{[-r / 2, r / 2]} & \text { if } r \text { is even, } \\ {[(-r+1) / 2,(r+1) / 2]} & \text { if } r \text { is odd. }\end{cases}
$$

Proof: There are two cases. 
Case: $r$ Even. By (39) and (38) the even polyphase component is $e_{0}(k)=e(2 k)$ for $-r \leq 2 k \leq r$, and both $e(-r)$ and $e(r)$ are nonzero by hypothesis 39 , so

$$
\text { supp_int }\left(e_{0}\right)=[-r / 2, r / 2] \text {. }
$$

The odd polyphase component is $e_{1}(k)=e(2 k-1)$ for $-r+1 \leq 2 k-1 \leq r-1$, but we don't know that $e$ is nonzero at the endpoints since they lie in the interior of supp_int $(e)$. For instance, Strang and Nguyen [7. Section 6.5] exhibit a family of dyadic WS wavelet filter banks, credited to Sweldens, whose lowpass filters have $h( \pm(r-1))=0$, and 37 provides another example. Thus, all we can conclude is that

$$
\operatorname{supp\_ int}\left(e_{1}\right) \subset[(-r+2) / 2, r / 2] .
$$

Combining (40) and (41) using (8) yields

$$
\begin{aligned}
\operatorname{supp} \_i n t(e) & =\operatorname{supp} \_\operatorname{int}\left(e_{0}\right) \vee \operatorname{supp} \_\operatorname{int}\left(e_{1}\right) \\
& =[-r / 2, r / 2] .
\end{aligned}
$$

Case: $r$ Odd. By (39) and (38) the even polyphase component is $e_{0}(k)=e(2 k)$ for $-r+1 \leq 2 k \leq r-1$, and $e$ may vanish at either endpoint since they both lie in the interior of supp_int $(e)$, so

$$
\operatorname{supp} \_\operatorname{int}\left(e_{0}\right) \subset[(-r+1) / 2,(r-1) / 2] \text {. }
$$

The odd polyphase component is $e_{1}(k)=e(2 k-1)$ for $-r \leq 2 k-1 \leq r$, and both $e(-r)$ and $e(r)$ are nonzero so

$$
\operatorname{supp} \_\operatorname{int}\left(e_{1}\right)=[(-r+1) / 2,(r+1) / 2] \text {. }
$$

Combining (43) and (44) using (8) yields

$$
\begin{aligned}
\operatorname{supp} \_i n t(e) & =\operatorname{supp} \_\operatorname{int}\left(e_{0}\right) \vee \operatorname{supp} \_i n t\left(e_{1}\right) \\
& =[(-r+1) / 2,(r+1) / 2] .
\end{aligned}
$$

Lemma 4: Suppose $E(z)$ is an odd-length FIR filter whose support interval is centered at -1 :

$$
\operatorname{supp\_ int}(e)=[-r-1, r-1], \quad r \equiv \operatorname{supp\_ rad}(e) \geq 0 \text {. }
$$

Then the polyphase support interval of the filter is:

$$
\operatorname{supp} \_\operatorname{int}(\boldsymbol{e})= \begin{cases}{[-r / 2, r / 2]} & \text { if } r \text { is even, } \\ {[(-r-1) / 2,(r-1) / 2]} & \text { if } r \text { is odd. }\end{cases}
$$

Proof: There are two cases.

Case: $r$ Even. The even polyphase component is $e_{0}(k)=e(2 k)$ for $-r \leq 2 k \leq r-2$, and $e$ may vanish at either endpoint since both lie in the interior of supp_int $(e)$, so

$$
\text { supp_int }\left(e_{0}\right) \subset[-r / 2,(r-2) / 2] \text {. }
$$

The odd polyphase component is $e_{1}(k)=e(2 k-1)$ for $-r-1 \leq 2 k-1 \leq r-1$, and $e_{1}(k) \neq 0$ at both endpoints so

$$
\operatorname{supp} \_i n t\left(e_{1}\right)=[-r / 2, r / 2] \text {. }
$$

Combining (47) and (48) using (8) yields

$$
\operatorname{supp} \_\operatorname{int}(\boldsymbol{e})=[-r / 2, r / 2] \text {. }
$$


Case: $r$ Odd. The even polyphase component is $e_{0}(k)=e(2 k)$ for $-r-1 \leq 2 k \leq r-1$, and $e$ is nonzero at both endpoints so

$$
\operatorname{supp} \_\operatorname{int}\left(e_{0}\right)=[(-r-1) / 2,(r-1) / 2] \text {. }
$$

The odd polyphase component is $e_{1}(k)=e(2 k-1)$ for $-r \leq 2 k-1 \leq r-2$, and $e$ may vanish at either endpoint since they both lie in the interior of supp_int $(e)$ so

$$
\operatorname{supp} \_ \text {int }\left(e_{1}\right) \subset[(-r+1) / 2,(r-1) / 2] .
$$

Combining (50) and (51) using (8) yields

$$
\operatorname{supp\_ int}(\boldsymbol{e})=[(-r-1) / 2,(r-1) / 2] \text {. }
$$

\section{The WS Support Interval Formula}

We now calculate the support intervals of the intermediate filters, $E_{i}^{(n)}(z)$, for irreducible lifting cascades in $\mathfrak{S}_{\mathcal{W}}$. Although stated for the case of interest-WS group lifting cascades- the next lemma only uses the support intervals for the filters and does not actually depend on the symmetry of either the intermediate WS filters or the HS lifting filters.

Lemma 5: Let $\mathbf{S}_{N-1}(z) \cdots \mathbf{S}_{0}(z) \in \mathcal{C}_{\mathcal{W}}$ be an irreducible cascade with intermediate scalar filters $E_{i}^{(n)}(z)$, $i=0,1$. Let $r_{i}^{(n)}$ be the support radius of $e_{i}^{(n)}$, and let $t^{(n)} \geq 1$ be the support radius of the HS lifting filter $S_{n}(z)$. Then supp_int $\left(e_{i}^{(n)}\right)$ is centered at $-i$,

$$
\operatorname{supp\_ int}\left(e_{i}^{(n)}\right)=\left[-r_{i}^{(n)}-i, r_{i}^{(n)}-i\right], \quad i=0,1,
$$

where

$$
\begin{gathered}
r_{m_{n}}^{(n)}=r_{1-m_{n}}^{(n)}+2 t^{(n)}-1 \quad \text { for } n \geq 0, \\
r_{1-m_{n}}^{(n)}=r_{m_{n}}^{(n-1)}+2 t^{(n-1)}-1 \quad \text { for } n \geq 1,
\end{gathered}
$$

with $r_{1-m_{0}}^{(0)}=r_{1-m_{0}}^{(-1)}=0$.

Proof: Induction on $n$.

Case: $n=0, m_{0}=0$. By (34) the lifting filter is centered at 1/2: supp_int $\left(s_{0}\right)=\left[-t^{(0)}+1, t^{(0)}\right]$. By (19) the lifted intermediate filter is

$$
E_{0}^{(0)}(z)=1+z S_{0}\left(z^{2}\right)
$$

and its complement is $E_{1}^{(0)}(z)=B_{1}(z)=z$, which implies $r_{1}^{(0)}=0$. Since $1=z^{0}$ we have supp_int $\left(z^{0}\right)=$ $\{0\}$. By 12 and (13)

$$
\begin{aligned}
\operatorname{supp} \_ \text {int }\left(z S_{0}\left(z^{2}\right)\right) & =\{-1\}+\left[2\left(-t^{(0)}+1\right), 2 t^{(0)}\right] \\
& =\left[-2 t^{(0)}+1,2 t^{(0)}-1\right] .
\end{aligned}
$$

Thus, $t^{(0)} \geq 1$ implies

$$
\operatorname{supp} \_\operatorname{int}\left(z^{0}\right) \subset\left(-2 t^{(0)}+1,2 t^{(0)}-1\right),
$$

which justifies applying formula (14) from Lemma 1 to (55):

$$
\begin{aligned}
\operatorname{supp} \_ \text {int }\left(e_{0}^{(0)}\right) & =\operatorname{supp} \_ \text {int }\left(z S_{0}\left(z^{2}\right)\right) \\
& =\left[-2 t^{(0)}+1,2 t^{(0)}-1\right] .
\end{aligned}
$$

We conclude that $r_{0}^{(0)}=2 t^{(0)}-1$, which verifies 53 since $r_{1}^{(0)}=0$. 
Case: $n=0, m_{0}=1$. By 35 the lifting filter is centered at $-1 / 2$ : $\operatorname{supp} \_$int $\left(s_{0}\right)=\left[-t^{(0)}, t^{(0)}-\right.$ 1]. By $[19)$ the lifted intermediate filter is

$$
E_{1}^{(0)}(z)=z+S_{0}\left(z^{2}\right)
$$

and its complement is $E_{0}^{(0)}(z)=B_{0}(z)=1$, which implies $r_{0}^{(0)}=0$. Since supp_int $(z)=\{-1\}$ and

$$
\text { supp_int }\left(S_{0}\left(z^{2}\right)\right)=\left[-2 t^{(0)}, 2\left(t^{(0)}-1\right)\right] \text {, }
$$

$t^{(0)} \geq 1$ implies

$$
\operatorname{supp} \_\operatorname{int}(z) \subset\left(-2 t^{(0)}, 2\left(t^{(0)}-1\right)\right),
$$

which justifies applying formula (14) to (56):

$$
\begin{aligned}
\operatorname{supp} \_ \text {int }\left(e_{1}^{(0)}\right) & =\operatorname{supp} \_ \text {int }\left(S_{0}\left(z^{2}\right)\right) \\
& =\left[-\left(2 t^{(0)}-1\right)-1,\left(2 t^{(0)}-1\right)-1\right] .
\end{aligned}
$$

We conclude that $r_{1}^{(0)}=2 t^{(0)}-1$, which verifies 53 since $r_{0}^{(0)}=0$.

Case: $n>0, m_{n}=0$. The case $m_{n}=0$ means that

$$
E_{1}^{(n)}(z)=E_{1}^{(n-1)}(z),
$$

and the induction hypothesis implies that supp_int $\left(e_{1}^{(n)}\right)$ is centered at -1 . The cascade is assumed irreducible so $E_{1}^{(n-1)}(z)$ must have been lifted in step $n-1$, and the induction hypothesis therefore implies

$$
r_{1}^{(n)}=r_{1}^{(n-1)}=r_{0}^{(n-1)}+2 t^{(n-1)}-1,
$$

which verifies (54). By (19) the lifted intermediate filter is

$$
E_{0}^{(n)}(z)=E_{0}^{(n-1)}(z)+S_{n}\left(z^{2}\right) E_{1}^{(n-1)}(z) .
$$

$S_{n}(z)$ is centered at $1 / 2$ so we have

$$
\operatorname{supp} \_\operatorname{int}\left(s_{n}\right)=\left[-t^{(n)}+1, t^{(n)}\right],
$$

and (12) and (13) give

$$
\begin{aligned}
& \operatorname{supp} \_ \text {int }\left(S_{n}\left(z^{2}\right) E_{1}^{(n-1)}(z)\right) \\
&= {\left[2\left(-t^{(n)}+1\right), 2 t^{(n)}\right]+\left[-r_{1}^{(n-1)}-1, r_{1}^{(n-1)}-1\right] } \\
& \quad=\left[-r_{1}^{(n-1)}-2 t^{(n)}+1, r_{1}^{(n-1)}+2 t^{(n)}-1\right] .
\end{aligned}
$$

Substitution of (57) into 59) gives

$$
\begin{aligned}
\operatorname{supp} \_ \text {int } & \left(S_{n}\left(z^{2}\right) E_{1}^{(n-1)}(z)\right) \\
= & {\left[-r_{0}^{(n-1)}-2\left(t^{(n-1)}+t^{(n)}-1\right),\right.} \\
& \left.r_{0}^{(n-1)}+2\left(t^{(n-1)}+t^{(n)}-1\right)\right] .
\end{aligned}
$$


The induction hypothesis says that supp_int $\left(e_{0}^{(n-1)}\right)$ is centered at 0 , with $t^{(n-1)} \geq 1$ and $t^{(n)} \geq 1$, so

$$
\begin{aligned}
\operatorname{supp} \_ \text {int }\left(e_{0}^{(n-1)}\right)= & {\left[-r_{0}^{(n-1)}, r_{0}^{(n-1)}\right] } \\
\subset & \left(-r_{0}^{(n-1)}-2\left(t^{(n-1)}+t^{(n)}-1\right),\right. \\
& \left.\quad r_{0}^{(n-1)}+2\left(t^{(n-1)}+t^{(n)}-1\right)\right) .
\end{aligned}
$$

This justifies applying (14) to (58):

$$
\operatorname{supp} \_ \text {int }\left(e_{0}^{(n)}\right)=\operatorname{supp} \_ \text {int }\left(S_{n}\left(z^{2}\right) E_{1}^{(n-1)}(z)\right) \text {. }
$$

Formula 59 implies that supp_int $\left(e_{0}^{(n)}\right)$ is centered at 0 and, since $r_{1}^{(n)}=r_{1}^{(n-1)}$, it verifies 53 :

$$
r_{0}^{(n)}=r_{1}^{(n)}+2 t^{(n)}-1 .
$$

Case: $n>0, m_{n}=1$. The case $m_{n}=1$ means that

$$
E_{0}^{(n)}(z)=E_{0}^{(n-1)}(z),
$$

and the induction hypothesis implies that supp_int $\left(e_{0}^{(n)}\right)$ is centered at 0 . The cascade is assumed irreducible so $E_{0}^{(n-1)}(z)$ must have been lifted in step $n-1$, and the induction hypothesis therefore implies

$$
r_{0}^{(n)}=r_{0}^{(n-1)}=r_{1}^{(n-1)}+2 t^{(n-1)}-1,
$$

which verifies (54). By (19) the lifted intermediate filter is

$$
E_{1}^{(n)}(z)=E_{1}^{(n-1)}(z)+S_{n}\left(z^{2}\right) E_{0}^{(n-1)}(z) .
$$

$S_{n}(z)$ is centered at $-1 / 2$ so we have

$$
\operatorname{supp} \_ \text {int }\left(s_{n}\right)=\left[-t^{(n)}, t^{(n)}-1\right],
$$

and (12) and (13) give

$$
\begin{aligned}
\operatorname{supp} \_ \text {int } & \left(S_{n}\left(z^{2}\right) E_{0}^{(n-1)}(z)\right) \\
= & {\left[-2 t^{(n)}, 2\left(t^{(n)}-1\right)\right]+\left[-r_{0}^{(n-1)}, r_{0}^{(n-1)}\right] } \\
= & {\left[-r_{0}^{(n-1)}-2 t^{(n)}, r_{0}^{(n-1)}+2 t^{(n)}-2\right] . }
\end{aligned}
$$

Substitution of 60 into 62 gives

$$
\begin{aligned}
\operatorname{supp} \_ \text {int } & \left(S_{n}\left(z^{2}\right) E_{0}^{(n-1)}(z)\right) \\
= & {\left[-r_{1}^{(n-1)}-2\left(t^{(n-1)}+t^{(n)}\right)+1,\right.} \\
& \left.r_{1}^{(n-1)}+2\left(t^{(n-1)}+t^{(n)}\right)-3\right] .
\end{aligned}
$$


The induction hypothesis says that supp_int $\left(e_{1}^{(n-1)}\right)$ is centered at -1 , with $t^{(n-1)} \geq 1$ and $t^{(n)} \geq 1$, so

$$
\begin{aligned}
\operatorname{supp} \_ \text {int }\left(e_{1}^{(n-1)}\right)= & {\left[-r_{1}^{(n-1)}-1, r_{1}^{(n-1)}-1\right] } \\
\subset & \left(-r_{1}^{(n-1)}-2\left(t^{(n-1)}+t^{(n)}\right)+1,\right. \\
& \left.\quad r_{1}^{(n-1)}+2\left(t^{(n-1)}+t^{(n)}\right)-3\right),
\end{aligned}
$$

which justifies applying (14) to 61):

$$
\operatorname{supp} \_ \text {int }\left(e_{1}^{(n)}\right)=\operatorname{supp} \_ \text {int }\left(S_{n}\left(z^{2}\right) E_{0}^{(n-1)}(z)\right) \text {. }
$$

Formula 62 implies that supp_int $\left(e_{1}^{(n)}\right)$ is centered at -1 and, since $r_{0}^{(n)}=r_{0}^{(n-1)}$, it verifies 53 :

$$
r_{1}^{(n)}=r_{0}^{(n)}+2 t^{(n)}-1
$$

\section{The Support-Covering Property}

Now we can prove that $\mathfrak{S}_{\mathcal{W}}$ satisfies the polyphase support-covering hypothesis 28 in Lemma 2 .

Lemma 6: Let $\mathbf{S}_{N-1}(z) \cdots \mathbf{S}_{0}(z) \in \mathcal{C}_{\mathcal{W}}$ be irreducible, with intermediate filters $E_{i}^{(n)}(z), i=0,1$. Then

$$
\operatorname{supp} \_ \text {int }\left(\boldsymbol{e}_{1-m_{n}}^{(n)}\right) \varsubsetneqq \operatorname{supp} \_ \text {int }\left(\boldsymbol{e}_{m_{n}}^{(n)}\right) \text { for } n \geq 0 .
$$

Proof: Let $r_{i}^{(n)}$ be the support radius of $e_{i}^{(n)}, i=0,1$. By Lemma 5 it follows that $r_{m_{n}}^{(n)}+r_{1-m_{n}}^{(n)}$ is odd, so $r_{0}^{(n)}$ and $r_{1}^{(n)}$ always have opposite parities.

Case: $r_{0}^{(n)}$ even, $r_{1}^{(n)}$ odd. By Lemma 3 and Lemma 4 the polyphase support intervals are:

$$
\begin{aligned}
& \text { supp_int }\left(\boldsymbol{e}_{0}^{(n)}\right)=\left[-r_{0}^{(n)} / 2, r_{0}^{(n)} / 2\right], \\
& \text { supp_int }\left(\boldsymbol{e}_{1}^{(n)}\right)=\left[-\left(r_{1}^{(n)}+1\right) / 2,\left(r_{1}^{(n)}-1\right) / 2\right] .
\end{aligned}
$$

If $m_{n}=0$ then applying Lemma 5 to 64 gives

$$
\begin{aligned}
& \operatorname{supp} \_ \text {int }\left(\boldsymbol{e}_{0}^{(n)}\right) \\
& =\left[-\left(r_{1}^{(n)}+2 t^{(n)}-1\right) / 2,\left(r_{1}^{(n)}+2 t^{(n)}-1\right) / 2\right] .
\end{aligned}
$$

Since $t^{(n)} \geq 1$, comparison with 65 yields

$$
\begin{gathered}
-\left(r_{1}^{(n)}+2 t^{(n)}-1\right) / 2 \leq-\left(r_{1}^{(n)}+1\right) / 2 \text { and } \\
\left(r_{1}^{(n)}+2 t^{(n)}-1\right) / 2>\left(r_{1}^{(n)}-1\right) / 2,
\end{gathered}
$$

which imply supp_int $\left(\boldsymbol{e}_{1}^{(n)}\right) \varsubsetneqq \operatorname{supp\_ int~}\left(\boldsymbol{e}_{0}^{(n)}\right)$.

If $m_{n}=1$ then applying Lemma 5 to 65 gives

$$
\begin{aligned}
& \operatorname{supp\_ int}\left(\boldsymbol{e}_{1}^{(n)}\right) \\
& \quad=\left[-\left(r_{0}^{(n)}+2 t^{(n)}\right) / 2,\left(r_{0}^{(n)}+2 t^{(n)}-2\right) / 2\right] .
\end{aligned}
$$


Since $t^{(n)} \geq 1$, comparison with 64 yields

$$
\begin{gathered}
-\left(r_{0}^{(n)}+2 t^{(n)}\right) / 2<-r_{0}^{(n)} / 2 \text { and } \\
\left(r_{0}^{(n)}+2 t^{(n)}-2\right) / 2 \geq r_{0}^{(n)} / 2,
\end{gathered}
$$

which imply supp_int $\left(\boldsymbol{e}_{0}^{(n)}\right) \varsubsetneqq \operatorname{supp} \_$int $\left(\boldsymbol{e}_{1}^{(n)}\right)$.

Case: $r_{0}^{(n)}$ odd, $r_{1}^{(n)}$ even. By Lemma 3 and Lemma 4 the polyphase support intervals are:

$$
\begin{aligned}
& \text { supp_int }\left(\boldsymbol{e}_{0}^{(n)}\right)=\left[-\left(r_{0}^{(n)}-1\right) / 2,\left(r_{0}^{(n)}+1\right) / 2\right], \\
& \text { supp_int }\left(\boldsymbol{e}_{1}^{(n)}\right)=\left[-r_{1}^{(n)} / 2, r_{1}^{(n)} / 2\right] .
\end{aligned}
$$

If $m_{n}=0$ then applying Lemma 5 to 66 gives

$$
\begin{aligned}
\operatorname{supp} \_ \text {int } & \left(\boldsymbol{e}_{0}^{(n)}\right) \\
= & {\left[-\left(r_{1}^{(n)}+2 t^{(n)}-2\right) / 2,\left(r_{1}^{(n)}+2 t^{(n)}\right) / 2\right] . }
\end{aligned}
$$

Since $t^{(n)} \geq 1$, comparison with 67 yields

$$
\begin{gathered}
-\left(r_{1}^{(n)}+2 t^{(n)}-2\right) / 2 \leq-r_{1}^{(n)} / 2 \text { and } \\
\left(r_{1}^{(n)}+2 t^{(n)}\right) / 2>r_{1}^{(n)} / 2,
\end{gathered}
$$

which implies supp_int $\left(\boldsymbol{e}_{1}^{(n)}\right) \varsubsetneqq \operatorname{supp} \_$int $\left(\boldsymbol{e}_{0}^{(n)}\right)$.

If $m_{n}=1$ then applying Lemma 5 to 67) gives

$$
\begin{aligned}
& \operatorname{supp\_ int}\left(\boldsymbol{e}_{1}^{(n)}\right) \\
& \quad=\left[-\left(r_{0}^{(n)}+2 t^{(n)}-1\right) / 2,\left(r_{0}^{(n)}+2 t^{(n)}-1\right) / 2\right] .
\end{aligned}
$$

Since $t^{(n)} \geq 1$, comparison with 66 yields

$$
\begin{gathered}
-\left(r_{0}^{(n)}+2 t^{(n)}-1\right) / 2<-\left(r_{0}^{(n)}-1\right) / 2 \text { and } \\
\left(r_{0}^{(n)}+2 t^{(n)}-1\right) / 2 \geq\left(r_{0}^{(n)}+1\right) / 2,
\end{gathered}
$$

which implies supp_int $\left(\boldsymbol{e}_{0}^{(n)}\right) \varsubsetneqq \operatorname{supp} \_$int $\left(\boldsymbol{e}_{1}^{(n)}\right)$.

\section{HALF-SAmple Symmetric Filter BANKS}

We briefly review the definitions from [1, Section IV-B] of the group lifting structures for the unimodular HS class, $\mathfrak{H}$. In the irreversible case, the scaling matrices are $\mathcal{D} \equiv\left\{\mathbf{D}_{K}: K \neq 0\right\}$. The groups of upper (respectively, lower) triangular lifting matrices, $\mathcal{U} \equiv v(\mathcal{P})$ (respectively, $\mathcal{L} \equiv \lambda(\mathcal{P})$ ), are defined by the same group of whole-sample antisymmetric real Laurent polynomials:

$$
\mathcal{P} \equiv\left\{S(z) \in \mathbb{R}\left[z, z^{-1}\right]: S\left(z^{-1}\right)=-S(z)\right\} .
$$

The set of concentric equal-length base HS filter banks defined in [1, Section IV-B] is

$$
\mathfrak{B}_{\mathfrak{H}} \equiv\left\{\mathbf{B} \in \mathfrak{H}: \operatorname{order}\left(B_{0}\right)=\operatorname{order}\left(B_{1}\right)\right\} .
$$


The lifting cascade group for the irreversible HS class is $\mathcal{C}_{\mathfrak{H}} \equiv\langle\mathcal{U} \cup \mathcal{L}\rangle$ and the irreversible HS group lifting structure is $\mathfrak{S}_{\mathfrak{H}} \equiv\left(\mathcal{D}, \mathcal{U}, \mathcal{L}, \mathfrak{B}_{\mathfrak{H}}\right)$.

There are no scaling matrices in the reversible case, $\mathcal{D}_{r} \equiv\{\mathbf{I}\}$, and the lifting filters 68 are restricted to those having dyadic rational coefficients. The set of base filter banks, $\mathfrak{B}_{\mathfrak{H}_{r}}$, for the reversible HS class is defined in [1, Section IV-B] to be the set of all matrices in $\mathfrak{B}_{\mathfrak{H}}$ that have dyadic lifting factorizations with no scaling matrices. Note that there are no other requirements (e.g., symmetry) for the dyadic lifting filters that factor reversible, concentric, equal-length HS base filter banks. The reversible HS class is defined to be

$$
\mathfrak{H}_{r} \equiv \mathcal{C}_{\mathfrak{H}_{r}} \mathfrak{B}_{\mathfrak{H}_{r}} \quad \text { where } \quad \mathcal{C}_{\mathfrak{H}_{r}} \equiv\left\langle\mathcal{U}_{r} \cup \mathcal{L}_{r}\right\rangle,
$$

and its group lifting structure is $\mathfrak{S}_{\mathfrak{H}_{r}} \equiv\left(\mathcal{D}_{r}, \mathcal{U}_{r}, \mathcal{L}_{r}, \mathfrak{B}_{\mathfrak{H}_{r}}\right)$.

\section{A. Main Uniqueness Result}

Our main result on HS filter banks shows that irreducible group lifting factorizations in $\mathfrak{S}_{\mathfrak{H}}$ are "almost" unique and that irreducible lifting factorizations in $\mathfrak{S}_{\mathfrak{H}_{r}}$ are unique.

Theorem 2 (Uniqueness of HS group lifting factorizations): Let $\mathfrak{S}_{\mathfrak{H}_{\mathfrak{H}}}$ and $\mathfrak{S}_{\mathfrak{H}_{r}}$ be the group lifting structures defined in [1, Section IV-B]. Every filter bank in $\mathfrak{H}$ has an irreducible group lifting factorization in $\mathfrak{S}_{\mathfrak{H}}$ that is unique modulo rescaling. Every filter bank in $\mathfrak{H}_{r}$ has a unique irreducible group lifting factorization in $\mathfrak{S}_{\mathfrak{H}_{r}}$.

Proof: Existence of irreducible group lifting factorizations for these classes was discussed in [1, Section IV-B].

Uniqueness: Let $\mathbf{B}(z) \in \mathfrak{B}_{\mathfrak{H}}$. By the definition of $\mathfrak{H}$ [1, Definition 9], both impulse responses are symmetric about $-1 / 2$ so 69 implies that $b_{0}$ and $b_{1}$ have the same scalar support intervals. By Lemma 7 (Section IV-B below), $\boldsymbol{b}_{0}$ and $\boldsymbol{b}_{1}$ have the same polyphase support intervals, verifying (27). Lemma 9 (Section IV-D below) shows that irreducible lifting cascades in $\mathcal{C}_{\mathfrak{H}} \mathfrak{B}_{\mathfrak{H}}$ satisfy (28), so Lemma 2 implies that $\mathfrak{S}_{\mathfrak{H}}$ is order-increasing. $\mathcal{D}$-invariance follows from [1, Lemma 2], so [1, Theorem 1] implies that irreducible lifting factorizations in $\mathfrak{S}_{\mathfrak{H}}$ are unique modulo rescaling.

In the reversible case, $\mathfrak{C}_{\mathfrak{H}_{r}}<\mathcal{C}_{\mathfrak{H}}$ and $\mathfrak{B}_{\mathfrak{H}_{r}} \subset \mathfrak{B}_{\mathfrak{H}}$. The reversible HS group lifting factorizations thus form a subset of the irreversible factorizations and are therefore unique modulo rescaling. However, given two irreducible factorizations of the same reversible filter bank,

$$
\mathbf{S}_{N-1}(z) \cdots \mathbf{S}_{0}(z) \mathbf{B}(z)=\mathbf{S}_{N-1}^{\prime}(z) \cdots \mathbf{S}_{0}^{\prime}(z) \mathbf{B}^{\prime}(z)
$$

we have $K^{\prime}=K=1$ since there are no scaling operations. Thus, $\mathbf{B}^{\prime}(z)=\mathbf{B}(z)$ and $\mathbf{S}_{i}^{\prime}(z)=\mathbf{S}_{i}(z)$ for all $i$, implying uniqueness of reversible HS group lifting factorizations.

Remarks: As in the WS case, Theorem 2 provides a more complete theory of both existence and uniqueness for cascade factorizations of HS filter banks than [5].

1) Applications: The JPEG 2000 standard does not contain source coding specifications for HS filter banks as a class, in part because of the complications inherent in their lifting factorizations. Instead, JPEG 2000 Part 2 [4, Annex H] contains specifications for lifting factorizations of arbitrary user-defined FIR filter banks, with no assumptions about the symmetries of either the filters or their lifting factors. Part 2 Annex H does contain a number of examples of HS filter banks, however, and we can use Theorem 2 to answer some questions about lifting factorization of HS filter banks that arose on the ISO committee during the writing of Part 2.

First let us address the filter bank normalization specifications in JPEG 2000 Part 2. We mentioned in Section [-C] that [1, Theorem 1] yields unique irreducible factorizations if one normalizes a degree of freedom in the base filter banks, e.g., by fixing a common nonzero value for $B_{0}(1)$. As explained in [8], 
JPEG 2000 Part 2 requires the normalization $H_{0}(1)=1$, a similar constraint. The JPEG 2000 constraint does not imply uniqueness, however. Let

$$
\mathbf{H}(z)=\mathbf{D}_{K} \mathbf{S}_{N-1}(z) \cdots \mathbf{S}_{0}(z) \mathbf{B}(z)
$$

satisfy $H_{0}(1)=1$, and pick $K^{\prime} \neq K$. Define $\alpha \equiv K / K^{\prime}$ so that $\mathbf{D}_{K}=\mathbf{D}_{K^{\prime}} \mathbf{D}_{\alpha}$ and substitute in (70). By [1, eqn. (28)],

$$
\mathbf{H}(z)=\mathbf{D}_{K^{\prime}} \gamma_{\alpha} \mathbf{S}_{N-1}(z) \cdots \gamma_{\alpha} \mathbf{S}_{0}(z) \mathbf{B}^{\prime}(z),
$$

where $\mathbf{B}^{\prime}(z) \equiv \mathbf{D}_{\alpha} \mathbf{B}(z)$. This gain transfer between the scaling and base matrices leaves $\mathbf{H}(z)$ unchanged, and therefore does not violate the constraint $H_{0}(1)=1$. According to Theorem 2, though, this is the only way in which two irreducible HS group lifting factorizations of $\mathbf{H}(z)$ can differ.

As described in [1, Section I-A], the JPEG 2000 example filter bank in [4, Annex H.4.1.2.1] contains a lifting factorization of an irreversible 6-tap/10-tap HS wavelet filter bank, $\mathbf{H}_{6,10}(z)$, that is lifted from a concentric 6-tap/6-tap HS base filter bank, $\mathbf{B}_{6,6}(z)$, by a second-order WA lifting step:

$$
\mathbf{H}_{6,10}(z)=\mathbf{S}_{0}(z) \mathbf{B}_{6,6}(z) .
$$

Interestingly, although $\mathbf{H}_{6,10}(z)$ is irreversible, the scaling factor is trivial $(K=1)$, which is why the scaling matrix has been omitted from (71). Factorization (71) is unique modulo rescaling by Theorem 2 , which answers in the negative the question of whether $\mathbf{H}_{6,10}(z)$ can be lifted from the Haar filter bank by a clever choice of WA lifting filters. The same answer holds for the question of whether the 10-tap/18-tap HS filter bank in [4, Annex H.4.1.2.2], which is lifted from a 10-tap/10-tap HS base filter bank by a fourth-order WA lifting filter, can similarly be lifted from the Haar using WA lifting filters.

Theorem 2 also applies to the group lifting structure $\mathfrak{S}_{E L A S F}$ for the ELASF family of reversible HS filter banks lifted from the Haar, as defined by M. Adams [9], [10]. It was shown in [1, Example 6] that the ELASF filter banks form a subset of the reversible HS class, $\mathfrak{H}_{r}$, and that every group lifting factorization in $\mathfrak{S}_{E L A S F}$ is a factorization in $\mathfrak{S}_{\mathfrak{H}_{r}}$. Thus, irreducible group lifting factorizations in $\mathfrak{S}_{E L A S F}$ are unique.

The lemmas needed to verify hypothesis (28) of Lemma 2 occupy the remainder of Section IV.

\section{B. Polyphase Support Intervals}

Polyphase support intervals for FIR filters centered at $-1 / 2$ are sufficient to cover HS group lifting structures. The formulas do not depend on linear phase properties of the filters.

Lemma 7: Suppose $E(z)$ is an even-length FIR filter whose support interval is centered at $-1 / 2$ :

$$
\operatorname{supp} \_ \text {int }(e)=[-r, r-1] \text { for } r \equiv \operatorname{supp\_ rad}(e) \geq 1 .
$$

Then the polyphase support interval of the filter is:

$$
\operatorname{supp} \_\operatorname{int}(\boldsymbol{e})= \begin{cases}{[-r / 2, r / 2]} & \text { if } r \text { is even, } \\ {[-(r-1) / 2,(r-1) / 2]} & \text { if } r \text { is odd. }\end{cases}
$$

Proof: There are two cases. 
Case: $r$ Even. The even polyphase component is $e_{0}(k)=e(2 k)$ for $-r \leq 2 k \leq r-2$, and $e(-r) \neq 0$ while $e(r-2)$ may be zero, so

$$
\operatorname{supp\_ int}\left(e_{0}\right)=[-r / 2, n]
$$

for some $n$ with $-r / 2 \leq n \leq(r-2) / 2$.

The odd component is $e_{1}(k)=e(2 k-1)$ for $-r+1 \leq 2 k-1 \leq r-1$, and $e(r-1) \neq 0$ while $e(-r+1)$ may be zero, so

$$
\operatorname{supp} \_\operatorname{int}\left(e_{1}\right)=[n, r / 2]
$$

for some $n$ with $(-r+2) / 2 \leq n \leq r / 2$. Combining (73) and (74) using (8) yields

$$
\begin{aligned}
\operatorname{supp} \_i n t(e) & =\operatorname{supp} \_\operatorname{int}\left(e_{0}\right) \vee \operatorname{supp} \_i n t\left(e_{1}\right) \\
& =[-r / 2, r / 2] .
\end{aligned}
$$

Case: $r$ Odd. The even polyphase component is $e_{0}(k)=e(2 k)$ for $-r+1 \leq 2 k \leq r-1$, and $e(r-1) \neq 0$ while $e(-r+1)$ may be zero, so

$$
\operatorname{supp} \_\operatorname{int}\left(e_{0}\right)=[n,(r-1) / 2]
$$

for some $n$ with $(-r+1) / 2 \leq n \leq(r-1) / 2$.

The odd component is $e_{1}(k)=e(2 k-1)$ for $-r \leq 2 k-1 \leq r-2$, and $e(-r) \neq 0$ while $e(r-2)$ may be zero, so

$$
\operatorname{supp\_ int}\left(e_{1}\right)=[(-r+1) / 2, n]
$$

for some $n$ with $(-r+1) / 2 \leq n \leq(r-1) / 2$. Combining (76) and 77) using [8] yields

$$
\operatorname{supp\_ int}(\boldsymbol{e})=[-(r-1) / 2,(r-1) / 2] \text {. }
$$

\section{The HS Support Interval Formula}

As in the WS case, the HS support interval formula does not depend on linear phase properties of the filters.

Lemma 8: Let $\mathbf{S}_{N-1}(z) \cdots \mathbf{S}_{0}(z) \mathbf{B}(z) \in \mathcal{C}_{\mathfrak{H}} \mathfrak{B}_{\mathfrak{H}}$ be irreducible, with intermediate scalar filters $E_{i}^{(n)}(z)$, $i=0,1$. Let $r_{i}^{(n)}$ be the support radius of $e_{i}^{(n)}$, and let $t^{(n)} \geq 1$ be the support radius of the WA lifting filter $S_{n}(z)$. Then supp_int $\left(e_{i}^{(n)}\right)$ is centered at $-1 / 2$,

$$
\text { supp_int }\left(e_{i}^{(n)}\right)=\left[-r_{i}^{(n)}, r_{i}^{(n)}-1\right], \quad i=0,1,
$$

where

$$
\begin{gathered}
r_{m_{n}}^{(n)}=r_{1-m_{n}}^{(n)}+2 t^{(n)} \quad \text { for } n \geq 0, \\
r_{1-m_{n}}^{(n)}=r_{m_{n}}^{(n-1)}+2 t^{(n-1)} \quad \text { for } n \geq 1,
\end{gathered}
$$

with $r_{1-m_{0}}^{(0)}=r^{(-1)}$, the common support radius of both base filters, $b_{i}$.

Proof: Induction on $n$. In all cases, the WA lifting filter is centered at 0: $\operatorname{supp} \_\operatorname{int}\left(s_{n}\right)=\left[-t^{(n)}, t^{(n)}\right]$. The concentric equal-length base filter bank, $\mathbf{B}(z) \in \mathfrak{B}_{\mathfrak{H}}$, has both filters centered at $-1 / 2$ :

$$
\operatorname{supp} \_ \text {int }\left(b_{i}\right)=\left[-r^{(-1)}, r^{(-1)}-1\right], \quad i=0,1 \text {. }
$$


Case: $n=0 . \quad$ By (19) the lifted intermediate filter is

$$
E_{m_{0}}^{(0)}(z)=B_{m_{0}}(z)+S_{0}\left(z^{2}\right) B_{1-m_{0}}(z)
$$

and its complement is $E_{1-m_{0}}^{(0)}(z)=B_{1-m_{0}}(z)$, implying $r_{1-m_{0}}^{(0)}=r^{(-1)}$. By 12 and 13 ,

$$
\begin{aligned}
\operatorname{supp\_ int} & \left(S_{0}\left(z^{2}\right) B_{1-m_{0}}(z)\right) \\
= & {\left[-2 t^{(0)}, 2 t^{(0)}\right]+\left[-r^{(-1)}, r^{(-1)}-1\right] } \\
& =\left[-2 t^{(0)}-r^{(-1)}, 2 t^{(0)}+r^{(-1)}-1\right] .
\end{aligned}
$$

By $81, t^{(0)} \geq 1$ implies

$$
\operatorname{supp} \_\operatorname{int}\left(b_{m_{0}}\right) \subset\left(-2 t^{(0)}-r^{(-1)}, 2 t^{(0)}+r^{(-1)}-1\right),
$$

which justifies applying formula (14) from Lemma 1 to 82 :

$$
\begin{aligned}
\operatorname{supp} \_i n t\left(e_{m_{0}}^{(0)}\right) & =\operatorname{supp\_ int}\left(S_{0}\left(z^{2}\right) B_{1-m_{0}}(z)\right) \\
& =\left[-2 t^{(0)}-r^{(-1)}, 2 t^{(0)}+r^{(-1)}-1\right] .
\end{aligned}
$$

This shows that supp_int $\left(e_{m_{0}}^{(0)}\right)$ is centered at $-1 / 2$ with

$$
\operatorname{supp} \_ \text {rad }\left(e_{m_{0}}^{(0)}\right)=r^{(-1)}+2 t^{(0)},
$$

verifying (79).

Case: $n>0$. The complement of the lifted filter is

$$
E_{1-m_{n}}^{(n)}(z)=E_{1-m_{n}}^{(n-1)}(z)=E_{m_{n-1}}^{(n-1)}(z)
$$

by irreducibility. Use the induction hypothesis to apply $\sqrt{79}$ to $E_{m_{n-1}}^{(n-1)}(z)$ and note that $m_{n}=1-m_{n-1}$ :

$$
r_{1-m_{n}}^{(n)}=r_{1-m_{n}}^{(n-1)}=r_{m_{n-1}}^{(n-1)}=r_{m_{n}}^{(n-1)}+2 t^{(n-1)},
$$

which verifies (80). By (19) the lifted intermediate filter is

$$
E_{m_{n}}^{(n)}(z)=E_{m_{n}}^{(n-1)}(z)+S_{n}\left(z^{2}\right) E_{1-m_{n}}^{(n-1)}(z)
$$

supp_int $\left(e_{1-m_{n}}^{(n-1)}\right)$ is centered at $-1 / 2$ by induction, so 12 and 13 give

$$
\begin{aligned}
\operatorname{supp} \_i n t & \left(S_{n}\left(z^{2}\right) E_{1-m_{n}}^{(n-1)}(z)\right) \\
= & {\left[-2 t^{(n)}, 2 t^{(n)}\right]+\left[-r_{1-m_{n}}^{(n-1)}, r_{1-m_{n}}^{(n-1)}-1\right] } \\
= & {\left[-2 t^{(n)}-r_{1-m_{n}}^{(n-1)}, 2 t^{(n)}+r_{1-m_{n}}^{(n-1)}-1\right] . }
\end{aligned}
$$

Substitution of (83) into (85) gives

$$
\begin{aligned}
\operatorname{supp} \_ \text {int } & \left(S_{n}\left(z^{2}\right) E_{1-m_{n}}^{(n-1)}(z)\right) \\
= & {\left[-r_{m_{n}}^{(n-1)}-2\left(t^{(n-1)}+t^{(n)}\right),\right.} \\
& \left.r_{m_{n}}^{(n-1)}+2\left(t^{(n-1)}+t^{(n)}\right)-1\right] .
\end{aligned}
$$


The induction hypothesis says that supp_int $\left(e_{m_{n}}^{(n-1)}\right)$ is centered at $-1 / 2$, with $t^{(n-1)} \geq 1$ and $t^{(n)} \geq 1$, so

$$
\begin{aligned}
\operatorname{supp} \_ \text {int }\left(e_{m_{n}}^{(n-1)}\right)= & {\left[-r_{m_{n}}^{(n-1)}, r_{m_{n}}^{(n-1)}-1\right] } \\
\subset & \left(-r_{m_{n}}^{(n-1)}-2\left(t^{(n-1)}+t^{(n)}\right),\right. \\
& \left.\quad r_{m_{n}}^{(n-1)}+2\left(t^{(n-1)}+t^{(n)}\right)-1\right) .
\end{aligned}
$$

This justifies applying (14) to (84):

$$
\operatorname{supp} \_ \text {int }\left(e_{m_{n}}^{(n)}\right)=\operatorname{supp} \_ \text {int }\left(S_{n}\left(z^{2}\right) E_{1-m_{n}}^{(n-1)}(z)\right) \text {. }
$$

Formula 85 shows that supp_int $\left(e_{m_{n}}^{(n)}\right)$ is centered at $-1 / 2$, and substituting $r_{1-m_{n}}^{(n)}=r_{1-m_{n}}^{(n-1)}$ into 85 , verifies 79 :

$$
r_{m_{n}}^{(n)}=r_{1-m_{n}}^{(n)}+2 t^{(n)}
$$

\section{The Support-Covering Property}

We now prove that $\mathfrak{S}_{\mathfrak{H}}$ satisfies the polyphase support-covering hypothesis (28) in Lemma 2 ,

Lemma 9: Let $\mathbf{S}_{N-1}(z) \cdots \mathbf{S}_{0}(z) \mathbf{B}(z) \in \mathcal{C}_{\mathfrak{H}} \mathfrak{B}_{\mathfrak{H}}$ be irreducible, with intermediate filters $E_{i}^{(n)}(z), i=$ 0,1 . Then

$$
\operatorname{supp\_ int}\left(\boldsymbol{e}_{1-m_{n}}^{(n)}\right) \varsubsetneqq \operatorname{supp} \_ \text {int }\left(\boldsymbol{e}_{m_{n}}^{(n)}\right) \quad \text { for } n \geq 0 .
$$

Proof: Let $r_{i}^{(n)}$ be the support radius of $e_{i}^{(n)}, i=0,1$. By Lemma 8 it follows that $r_{m_{n}}^{(n)}+r_{1-m_{n}}^{(n)}$ is even, so $r_{0}^{(n)}$ and $r_{1}^{(n)}$ always have equal parities.

Case: $r_{0}^{(n)}, r_{1}^{(n)}$ even. Regardless of the value of $m_{n}$, Lemma 7 implies

$$
\begin{aligned}
& \operatorname{supp} \_i n t\left(\boldsymbol{e}_{m_{n}}^{(n)}\right)=\left[-r_{m_{n}}^{(n)} / 2, r_{m_{n}}^{(n)} / 2\right] \text {, } \\
& \operatorname{supp} \_ \text {int }\left(\boldsymbol{e}_{1-m_{n}}^{(n)}\right)=\left[-r_{1-m_{n}}^{(n)} / 2, r_{1-m_{n}}^{(n)} / 2\right] \text {. }
\end{aligned}
$$

Applying Lemma 8 to 87 gives

$$
\begin{aligned}
\operatorname{supp} \_ \text {int } & \left(\boldsymbol{e}_{m_{n}}^{(n)}\right) \\
& =\left[-\left(r_{1-m_{n}}^{(n)}+2 t^{(n)}\right) / 2,\left(r_{1-m_{n}}^{(n)}+2 t^{(n)}\right) / 2\right] .
\end{aligned}
$$

Since $t^{(n)} \geq 1$, comparison with 88 shows that

$$
\operatorname{supp} \_ \text {int }\left(\boldsymbol{e}_{1-m_{n}}^{(n)}\right) \varsubsetneqq \operatorname{supp} \_ \text {int }\left(\boldsymbol{e}_{m_{n}}^{(n)}\right) \text {. }
$$


Case: $r_{0}^{(n)}, r_{1}^{(n)}$ odd. Regardless of the value of $m_{n}$, Lemma 7 implies

$$
\begin{gathered}
\operatorname{supp\_ int}\left(\boldsymbol{e}_{m_{n}}^{(n)}\right) \\
=\left[-\left(r_{m_{n}}^{(n)}-1\right) / 2,\left(r_{m_{n}}^{(n)}-1\right) / 2\right], \\
\text { supp_int }\left(\boldsymbol{e}_{1-m_{n}}^{(n)}\right) \\
=\left[-\left(r_{1-m_{n}}^{(n)}-1\right) / 2,\left(r_{1-m_{n}}^{(n)}-1\right) / 2\right] .
\end{gathered}
$$

Applying Lemma 8 to 89 , gives

$$
\begin{aligned}
& \operatorname{supp} \_ \text {int }\left(\boldsymbol{e}_{m_{n}}^{(n)}\right) \\
& \quad=\left[-\left(r_{1-m_{n}}^{(n)}+2 t^{(n)}-1\right) / 2,\left(r_{1-m_{n}}^{(n)}+2 t^{(n)}-1\right) / 2\right] .
\end{aligned}
$$

Since $t^{(n)} \geq 1$, comparison with 90 shows that

$$
\operatorname{supp\_ int}\left(\boldsymbol{e}_{1-m_{n}}^{(n)}\right) \varsubsetneqq \operatorname{supp} \_ \text {int }\left(\boldsymbol{e}_{m_{n}}^{(n)}\right) \text {. }
$$

\section{CONCLusions}

The theory of group lifting structures developed in [1] has been used to prove uniqueness of linear phase lifting factorizations for the two nontrivial classes of linear phase two-channel perfect reconstruction filter banks, the WS and HS classes. A key technical result, Lemma 2, provides sufficient conditions for inferring that a group lifting structure satisfies the critical polyphase order-increasing property defined in [1]. In the WS case, Theorem 1 states that both reversible and irreversible WS filter banks have unique irreducible group lifting factorizations using HS lifting filters. Theorem 1 implies, e.g., that left matrix factorizations of WS filter banks into HS lifting filters are identical to right matrix factorizations. The scope of Theorem 1 covers the specifications for WS filter banks in Part 2 Annex G of the ISO/IEC JPEG 2000 standard.

In the HS case, Theorem 2 covers liftings from equal-length HS base filter banks using WA lifting filters. In the irreversible case, the WA lifting filters and the equal-length HS base filter banks are unique modulo rescaling. This implies, for instance, that 6-tap/10-tap and 10-tap/18-tap HS filter banks specified in JPEG 2000 Part 2 Annex H cannot be lifted from the Haar filter bank using WA lifting filters. Group lifting factorizations of reversible HS filter banks are unique, including M. Adams' ELASF class of reversible HS filter banks lifted from the Haar. A follow-on paper will characterize the structure of the groups associated with group lifting structures that satisfy the hypotheses of the uniqueness theorem.

\section{REFERENCES}

[1] C. M. Brislawn, "Group lifting structures for multirate filter banks, I: Uniqueness of lifting factorizations," Los Alamos National Lab, Tech. Rep. LAUR-09-2983, May 2009, to appear in IEEE Trans. Signal Process.

[2] C. M. Brislawn and B. Wohlberg, "The polyphase-with-advance representation and linear phase lifting factorizations," IEEE Trans. Signal Process., vol. 54, no. 6, pp. 2022-2034, Jun. 2006.

[3] Information technology_JPEG 2000 Image Coding System, Part 1, ser. ISO/IEC Int'1. Standard 15444-1, ITU-T Rec. T.800. Int'l. Org. for Standardization, Dec. 2000.

[4] Information technology—JPEG 2000 Image Coding System, Part 2 (Extensions), ser. ISO/IEC Int'l. Standard 15444-2, ITU-T Rec. T.801. Int'l. Org. for Standardization, May 2004.

[5] T. Q. Nguyen and P. P. Vaidyanathan, "Two-channel perfect-reconstruction FIR QMF structures which yield linear-phase analysis and synthesis filters," IEEE Trans. Acoust., Speech, Signal Process., vol. 37, no. 5, pp. 676-690, May 1989. 
[6] D. S. Taubman and M. W. Marcellin, JPEG2000: Image Compression Fundamentals, Standards, and Practice. Boston, MA: Kluwer, 2002.

[7] G. Strang and T. Nguyen, Wavelets and Filter Banks. Wellesley, MA: Wellesley-Cambridge, 1996.

[8] C. M. Brislawn and B. Wohlberg, "Gain normalization of lifted filter banks," Signal Processing, vol. 87, no. 6, pp. 12811287, Jun. 2007.

[9] M. D. Adams, "Reversible integer-to-integer wavelet transforms for image coding," Ph.D. dissertation, University of British Columbia, Vancouver, BC, Canada, Sep. 2002.

[10] M. D. Adams and R. K. Ward, "Symmetric-extension-compatible reversible integer-to-integer wavelet transforms," IEEE Trans. Signal Process., vol. 51, no. 10, pp. 2624-2636, Oct. 2003. 